\title{
Symptomatic Nephroptosis (SN) Causes the Loin Pain and Haematuria Syndrome (LPHS)
}

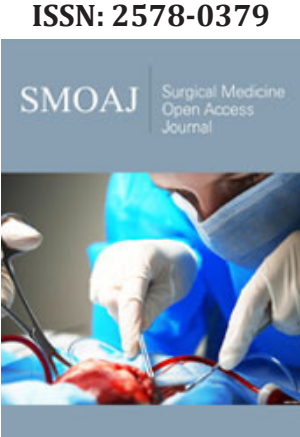

*Corresponding author: Ahmed N Ghanem, Department of Urology, Egypt

Submission: 海侐 January 21, 2020

Published: 海 February 10, 2020

Volume 3 - Issue 3

How to cite this article: Ahmed N Ghanem. Symptomatic Nephroptosis (SN) Causes the Loin Pain and Haematuria Syndrome (LPHS). Surg Med Open Acc J.3(3). SMOAJ.000562.2020. DOI: 10.31031/SMOAJ.2020.03.000562.

Copyright@ Ahmed N Ghanem, This article is distributed under the terms of the Creative Commons Attribution 4.0 International License, which permits unrestricted use and redistribution provided that the original author and source are credited.

\author{
Ahmed N Ghanem* \\ Department of Urology, Egypt
}

Keywords: LPHS; Nephroptosis; Nephropexy; IVU; RGP; Sympathetic denervation

Abbreviations: LPHS: Loin Pain Hematuria Syndrome; IVU: Intravenous Urography; RGP: Retrograde Pyelography

\section{Letter to Editor}

The objective of this letter is to report recent advances on the diagnosis and therapy of the loin pain hematuria syndrome (LPHS). The patho-aetiological link of LPHS with symptomatic nephroptosis (SN) is a novel discovery of the $21^{\text {st }}$ century that was first preliminary reported in 2002 [1]. The link was confirmed in 2016 in an article that précised the exact pathoaetiology of LPHS; ischemic nephritis and sympathetic neuropathy of SN. It also advanced a $100 \%$ curative therapy for LPHS namely; the renal sympathetic denervation and nephropexy (RSD\&N) surgery [2]. Symptomatic nephroptosis has been known for centuries [3] but was disparaged [4] >70 years ago and omitted from all textbooks. Hence it is a forgotten disease. The loin pain hematuria syndrome was first reported in 1967 [5] and has remained without any effective therapy till now [6-8].

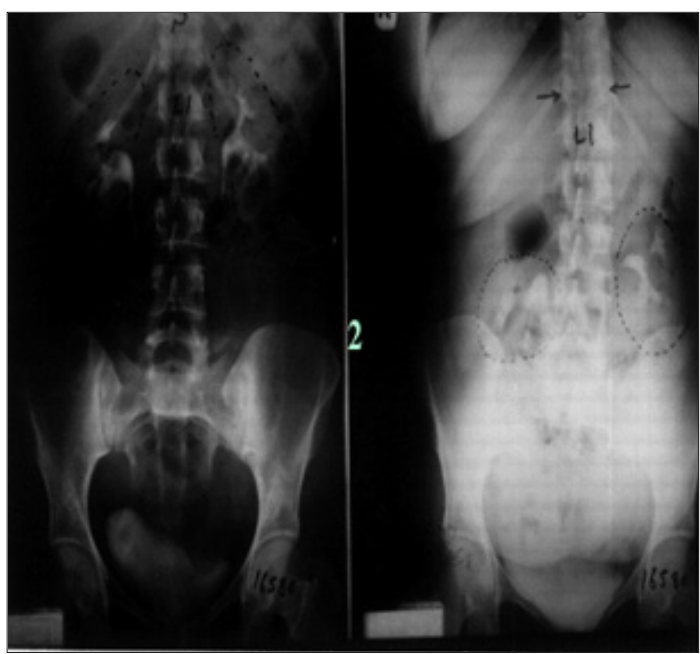

Figure 1: Compares IVU supine with IVU-E films shows bilateral nephroptosis of 3 right and 2 left vertebrae. Unilateral nephroptosis is the commonest affecting only the right side.

The discovery of SN causing LPHS and the successful curable therapy are based on a prospective cohort 10 years study [1,2] of 190 patients diagnosed with SN among whom $36(18.9 \%)$ patients developed LPHS. Two new signs that demonstrate renal pedicle stretch of SN (Figure 1) causes vessel stenosis and ischemic nephritis as evidenced by the new IVU7 sign and tube stretch hypothesis (Figure $2 \& 3$ ). These signs demonstrate that stretching of renal artery in pedicle at erect posture causes vessels stenosis, diminished blood flow and induces ischemic nephritis and sympathetic neuropathy. This is illustrated by producing the same effect on stretching a rubber catheter tube causing stenosis (reduced diameter) that diminishes lumen flow. Lumen flow of the blood vessel or catheter is completely obliterated 
when stretch of more than double the length of vessel or tube is accompanied by rotation twist. Ischemic nephritis affects the renal medulla long before the cortex, particularly eroding the medullary papillae creating fistulae between renal blood vessels and the collecting system explaining the venous shunt causing the hematuria. This is demonstrated only on retrograde pyelography (RGP) (Figure $4 \& 5$ ). So, the diagnosis of SN is only possible on intravenous urography (IVU) with erect film (IVU-E) on which the IVU7 sign demonstrates renal drop and pedicle stretch (Figure 1 \& 2) and RGP that demonstrates medullary damage (Figure 4 \& 5) while all other imaging including CAT and MRI are useless being feasible only on supine posture.

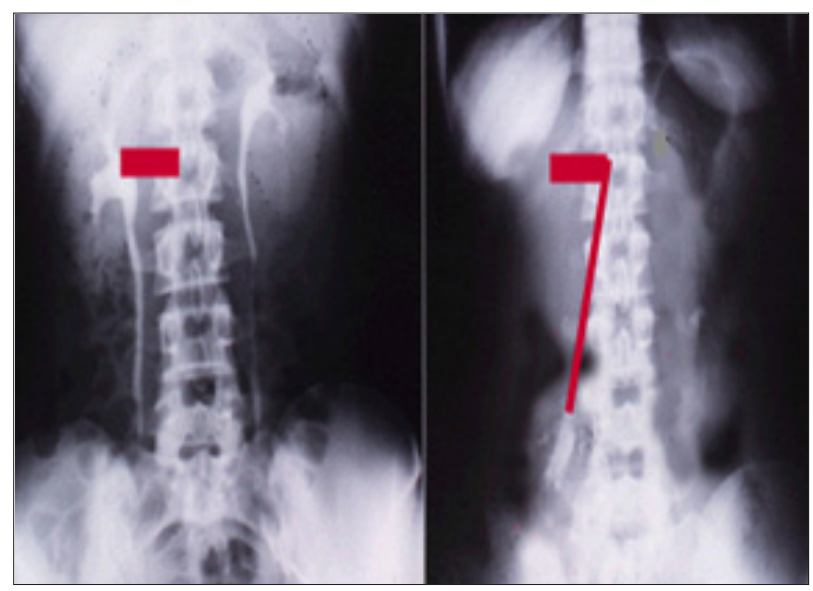

Figure 2: Shows renal pedicle mapped on a supine IVU film (Horizontal line segment, red) and the erect film (diagonal line segment, red) producing the figure of 7 where the renal pedicle is stretched to 3 times its normal length, causing vessels stenosis, reduction/cessation of blood flow and severe renal ischemia and sympathetic neuropathy.
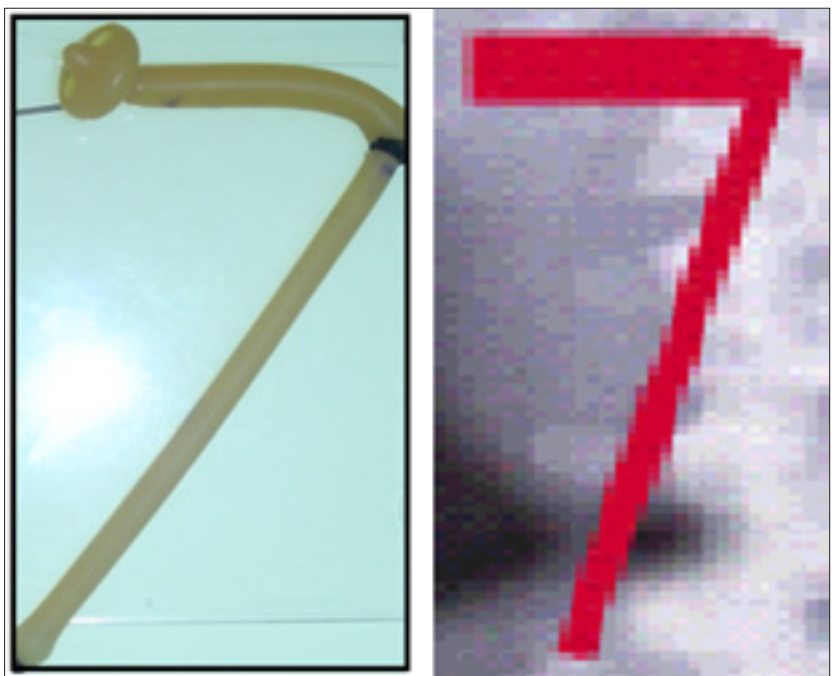

Figure 3: Compares the rubber tube stretch with IVU7 sign which demonstrate that stretch of a tube or artery causes stenosis (reduced diameter) thus reducing lumen flow.

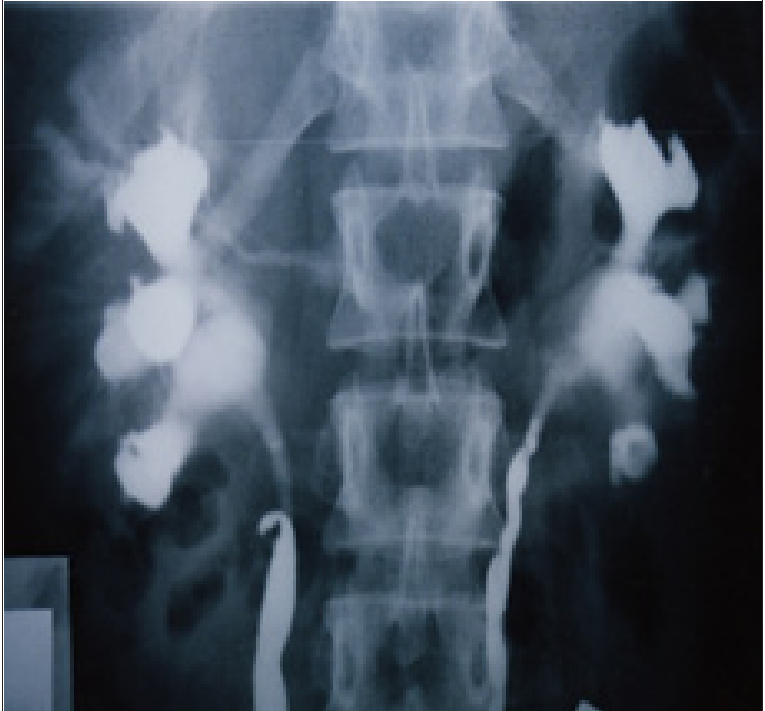

Figure 4: Shows RGP with multiple pyelocalyctaisis with erosion of medullary papillae and contrast leakage into veins (papillary fistulae connecting the collecting urinary system to veins i.e. venous shunt which is the site of hematuria

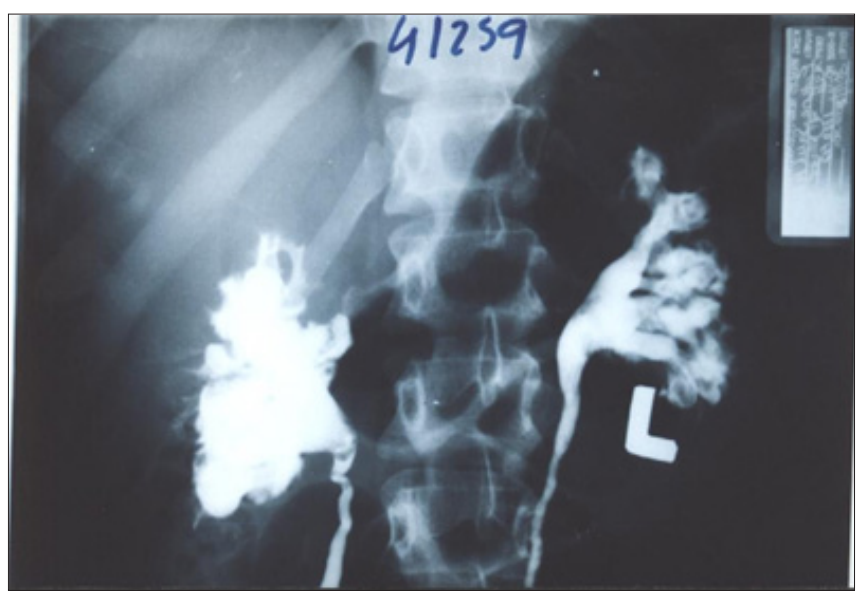

Figure 5: Shows advanced severe renal medullary papillary destruction in chronic long standing LPHS more severe on the right side in a long standing SN patient

Open surgical treatment was used in 28 patients suffering from severe LPHS complicating SN [2]; 10 had simple nephropexy and 18 had RSD\&N. Four of the 10 patients treated with simple nephropexy had recurrence of LPHS while those who had RSD\&N were all $100 \%$ cured of LPHS. Nephropexy [9] alone is inadequate treatment. Also renal sympathetic denervation alone is ineffective $[8,10]$. The two procedures must be done together and sympathetic denervation must be done on the renal pedicle, not anywhere else as chemical and lumbar sympathectomy do not work [8]. The evidence that repeated stretch of renal pedicle of SN causes ischemic focal infarctions of the kidney cortex at an advanced stage is shown in (Figure 6) as a result of the severe vessels stenosis (Figure 7) due to chronic stretch of the renal pedicle. Vessel stenosis is initially 
recurrent and intermittent occurring on erect posture but with chronicity of the disease it becomes fixed and permanent stenosis [5] as shown in Figure 7.

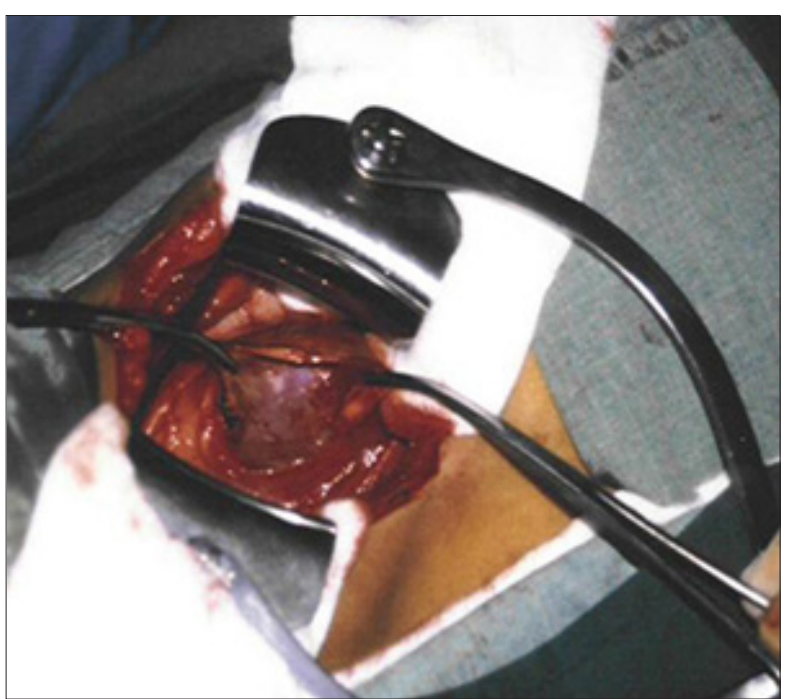

Figure 6: Shows an explored right kidney of chronic LPHS patient which is cyanotic with focal infarctions

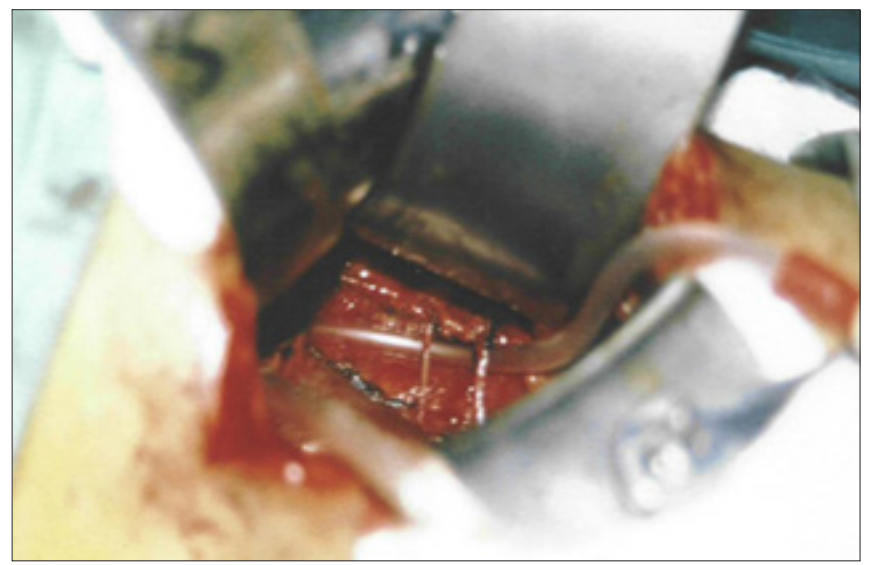

Figure 7: shows an explored right renal pedicle after denervation of the LPHS patient shown in Figure 6 with severe stenosis of artery and vein (effect of chronic repeated pedicle stretch). The Nelaton tube is approximately the size of a normal renal artery
Based on the above outlined discoveries on the diagnosis and therapy of LPHS it is strongly recommended that all concerned Physicians, Urologists and Nephrologists should implement it and report their own experience. It is also highly recommended that SN should be restored into modern textbooks of Surgery, Urology and Nephrology for which IVU-E and RGP are the recommended investigations making use of the new IVU7 sign to assess pedicle stretch and vessel stenosis inducing renal ischemia and sympathetic neuropathy causing LPHS. It is certain that this will help thousands of young patients worldwide, mostly females in their twenties and thirties, to get rid of their severe pain and hematuria of LPHS making their life miserable thus restoring their wellbeing, comfort and happiness again.

\section{Conflict of Interest}

None declared by the author. Funds received for the investigation and reporting the article: None.

\section{References}

1. Ghanem AN (2002) Features and complications of symptomatic nephroptosis causing the loin pain hematuria syndrome: Preleminary report. Saudi Med J 23(2): 197-205.

2. Ghanem SA, Ghanem AN (2016) Prospective observational study on loin pain hematuria syndrome complicating symptomatic nephroptosis and the results of renal sympathetic denervation and nephropexy surgery. J J Nephro Urol 3(1): 24.

3. Burford CE (1946) Nephroptosis with coexisting renal lesions. J Urol 55: 220-224.

4. Hoenig DM, Hemal AK, Shalhav AL, Clayman RV (1999) Nephroptosis: A disparaged condition revisited. Urology 54(4): 590-596.

5. Little PJ, Sloper JS, de Wardner HE (1967) A syndrome of loin pain hematuria associated with disease of the peripheral renal arteries. Q J Med 36(142): 253-259.

6. Armstrong T, McLean AD, Hayes M, Morgan BT, Tullock DN (2000) Early experience of intrauterine capsaicin infusion in loin pain hematuria syndrome. Br J Urol 85(3): 233-237.

7. (1992) Loin pain hematuria syndrome. Lancet 340 (2): 701-702.

8. Andrews BT, Jones NF, Browse NL (1997) The use of surgical sympathectomy in the treatment of chronic renal pain. Br J Urol 80(1): 6-10.

9. Hahn E (1881) Die operative Behandlung der beweglichen Niere Durch fixation. Zintralbl Chir 8: 449-452.

10. Blacklock AR (1989) Renal denervation with releasing renal capsule incision in the loin pain haematuria syndrome. Br J Urol 64(2): 203-204.

For possible submissions Click below: 